\title{
NOTE ON A LIMIT-POINT CRITERION
}

\author{
IAN KNOWLES
}

Abstract. A sufficient condition is given for the formal differential operator $\tau y(t)=\left(p(t) y^{\prime}(t)\right)^{\prime}+q(t) y(t)$ defined on the interval $[a, b), b \leqq \infty$, to be of limit-point type at $b$; this generalizes a criterion of Ismagilov given for the case $p(t)=1$ and $b=\infty$.

We consider the linear second order differential operator $\tau$ defined on the real half-open interval $[a, b), b \leqq \infty$, by

$$
\tau y(t)=\left(p(t) y^{\prime}(t)\right)^{\prime}+q(t) y(t)
$$

where $p^{-1}(t)>0$ and $q(t)$ are real-valued functions locally Lebesgue integrable on $[a, b)$. The operator $\tau$ is said to be of limit-circle type at $b$ if every solution $f(t)$ of the differential equation $\tau y(t)=0$ satisfies the condition

$$
\int_{a}^{b}\left|f^{2}(t)\right| d t<\infty
$$

If this is not the case, then $\tau$ is said to be of limit-point type at $b$.

In [1], Ismagilov proved that if $p(t)=1$ and $q(t) \leqq-q_{n}<0$ on disjoint intervals $I_{n} \subset[a, \infty)$ of length $\mu_{n}$ and $\sum_{n=1}^{\infty} q_{n}^{1 / 2} \mu_{n}^{3}=\infty$ then $\tau$ is of limitpoint type at $\infty$. We present here the corresponding result for the general operator $\tau$ defined on an arbitrary half-open subinterval of the real line.

THEOREM. If there exist finite disjoint subintervals $I_{n}, n=1,2, \cdots$, of $[a, b)$ and corresponding numbers $q_{n}$ and $p_{n}$ such that $q(t) \leqq-q_{n}<0$ and $p(t) \geqq p_{n}>0$ on $I_{n}$ and

$$
\sum_{n=1}^{\infty} p_{n}^{3 / 2} q_{n}^{1 / 2}\left(\int_{I_{n}} p^{-1}(s) d s\right)^{3}=\infty
$$

then the operator $\tau$ defined by equation (1) is of limit-point type at $b$.

Proof. For each $n=1,2, \cdots$, let the interval $I_{n}$ have endpoints $a_{n}$ and $b_{n}$, where $a_{n}<b_{n}$, and let $P(s)=\int_{a}^{s} p^{-1}(u) d u$ for any $s \in[a, b)$. We define the function $h(s)$ on $\bigcup_{n} I_{n}$ by

$$
h(s)=\left(P\left(b_{n}\right)-P\left(a_{n}\right)\right)^{-2}\left(P(s)-P\left(a_{n}\right)\right)^{2}\left(P\left(b_{n}\right)-P(s)\right)^{2}
$$

Received by the editors January 30, 1973.

AMS (MOS) subject classifications (1970). Primary 34B25.

Key words and phrases. Differential operator, second order, limit-point type.

c) American Mathematical Society 1973 
for $s \in I_{n}$. Then $h\left(a_{n}\right)=h\left(b_{n}\right)=h^{\prime}\left(a_{n}\right)=h^{\prime}\left(b_{n}\right)=0$ and, on each interval $I_{n}$,

$$
\begin{aligned}
& p(s)\left(p(s) h^{\prime}(s)\right)^{\prime} \\
& =2\left(P\left(b_{n}\right)-P\left(a_{n}\right)\right)^{-2}\left[6 P^{2}(s)-6 P(s)\left(P\left(b_{n}\right)+P\left(a_{n}\right)\right)\right. \\
& \left.+P^{2}\left(a_{n}\right)+4 P\left(a_{n}\right) P\left(b_{n}\right)+P^{2}\left(b_{n}\right)\right] \leqq 2
\end{aligned}
$$

because this is a quadratic function in the variable $P(s)$ which takes its maximum value at either endpoint of $I_{n}$. Also, after integrating by parts twice, it is easily seen that for any solution $f(s)$ of $\tau y(s)=0$,

$$
\int_{I_{n}} f^{2}(s)\left(p(s) h^{\prime}(s)\right)^{\prime} d s=2 \int_{I_{n}} h(s)\left[p(s)\left(f^{\prime}(s)\right)^{2}-q(s) f^{2}(s)\right] d s .
$$

Now let $u(s)$ and $v(s)$ be any two independent real-valued solutions of $\tau y(s)=0$ for which $p\left(u v^{\prime}-u^{\prime} v\right)=1$. Then, on each interval $I_{n}$,

$$
\begin{aligned}
2(-q)^{1 / 2} p^{-1 / 2} & =2(-q)^{1 / 2} u\left(p^{1 / 2} v^{\prime}\right)-2(-q)^{1 / 2} v\left(p^{1 / 2} u^{\prime}\right) \\
& \leqq p\left[\left(u^{\prime}\right)^{2}+\left(v^{\prime}\right)^{2}\right]-q\left[u^{2}+v^{2}\right] .
\end{aligned}
$$

Hence

$$
\begin{aligned}
\int_{a}^{b}\left(u^{2}+v^{2}\right) d s \geqq & \sum_{n=1}^{\infty} \int_{I_{n}}\left(u^{2}+v^{2}\right) d s \\
\geqq & \sum_{n=1}^{\infty}\left[\sup _{I_{n}} p\left(p h^{\prime}\right)^{\prime}\right]^{-1} \int_{I_{n}}\left(u^{2}+v^{2}\right) p\left(p h^{\prime}\right)^{\prime} d s \\
\geqq & \sum_{n=1}^{\infty} p_{n}\left[\sup _{I_{n}} p\left(p h^{\prime}\right)^{\prime}\right]^{-1} \int_{I_{n}}\left(u^{2}+v^{2}\right)\left(p h^{\prime}\right)^{\prime} d s \\
= & 2 \sum_{n=1}^{\infty} p_{n}\left[\sup _{I_{n}} p\left(p h^{\prime}\right)^{\prime}\right]^{-1} \\
& \times \int_{I_{n}} h\left[p\left(\left(u^{\prime}\right)^{2}+\left(v^{\prime}\right)^{2}\right)-q\left(u^{2}+v^{2}\right)\right] d s \quad \text { from (6) } \\
\geqq & 4 \sum_{n=1}^{\infty} p_{n}\left[\sup _{I_{n}} p\left(p h^{\prime}\right)^{\prime}\right]^{-1} \int_{I_{n}} h(-q)^{1 / 2} p^{-1 / 2} d s \quad \text { from (7) } \\
\geqq & 4 \sum_{n=1}^{\infty} q_{n}^{1 / 2} p_{n}^{3 / 2}\left[\sup _{I_{n}} p\left(p h^{\prime}\right)^{\prime}\right]^{-1} h(s) p^{-1}(s) d s \\
\geqq & 2 \sum_{n=1}^{\infty} q_{n}^{1 / 2} p_{n}^{3 / 2} \int_{I_{n}} h(s) p^{-1}(s) d s \text { using the property (5) } \\
= & \frac{1}{15} \sum_{n=1}^{\infty} q_{n}^{1 / 2} p_{n}^{3 / 2}\left(\int_{I_{n}} p^{-1}(s) d s\right)^{3}=\infty .
\end{aligned}
$$

This completes the proof. Q.E.D. 
At this stage, one might reasonably expect to improve this theorem by using another function $g(s)$ satisfying

$$
g\left(a_{n}\right)=g\left(b_{n}\right)=g^{\prime}\left(a_{n}\right)=g^{\prime}\left(b_{n}\right)=0, \quad n=1,2, \cdots,
$$

in the proof, instead of the function $h(s)$ defined by (4). Unfortunately, no such improvement is possible. To see this, let

$$
T_{n}(g)=\left[\sup _{I_{n}} p\left(p g^{\prime}\right)^{\prime}\right]^{-1} \int_{I_{n}} g(s) p^{-1}(s) d s,
$$

and substitute $g(s)$ for $h(s)$ in the preceding proof; the inequality (8) can now be rewritten in the form

$$
\int_{a}^{b}\left(u^{2}+v^{2}\right) d s \geqq 4 \sum_{n=1}^{\infty} q_{n}^{1 / 2} p_{n}^{3 / 2} T_{n}(g)
$$

which shows that $\tau$ is of limit-point type at $b$ if we have $\sum_{n=1}^{\infty} q_{n}^{1 / 2} p_{n}^{3 / 2} T_{n}(g)=$ $\infty$. But,

$$
\begin{aligned}
T_{n}(g) & =\frac{1}{2}\left[\sup _{I_{n}} p\left(p g^{\prime}\right)^{\prime}\right]^{-1} \int_{I_{n}}\left(P(s)-P\left(a_{n}\right)\right)^{2}\left(p(s) g^{\prime}(s)\right)^{\prime} d s \\
& \leqq \frac{1}{2} \int_{I_{n}}\left(P(s)-P\left(a_{n}\right)\right)^{2} p^{-1}(s) d s=\frac{1}{6}\left(\int_{I_{n}} p^{-1}(s) d s\right)^{3}
\end{aligned}
$$

and this criterion is therefore only a special case of the theorem. Thus, choosing another function $g(s)$ can at best only improve the constant appearing in the inequality (9).

\section{REFERENCE}

1. R. S. Ismagilov, On the self-adjointness of the Sturm-Liouville operator, Uspehi Mat. Nauk 18 (1963), no. 5 (113), 161-166. (Russian) MR 27 \#4979.

School of Mathematical Sciences, Flinders University of South Australia, Bedford Park, South Australia

DEPARTMENT OF MATHEMATICS, UNIVERSITY OF THE WITWATERSRAND, JOHANNESBURG, SoUTH AFrICA (Current address) 